\title{
DEL TIEMPO HISTÓRICO A LA CONCIENCIA HISTÓRICA: CAMBIOS EN LA ENSEÑANZA Y EL APRENDIZAJE DE LA HISTORIA EN LOS ÚLTIMOS 25 AÑOS
}

\author{
FROM HISTORICAL TIME TO HISTORICAL CONSCIOUSNESS: CHANGES \\ IN THE TEACHING AND LEARNING OF HISTORY IN THE LAST 25 YEARS
}

\author{
Antoni Santisteban Fernández*
}

\begin{abstract}
Este artículo repasa la relación que ha existido en los últimos 25 años entre las investigaciones acerca del aprendizaje de la temporalidad y la enseñanza de la historia. Los trabajos pioneros de la psicología evolutiva fueron aplicados de forma mecánica a la enseñanza de la historia. Su posterior revisión dio lugar a nuevas propuestas desde la didáctica de la historia. En la actualidad estas perspectivas se integran en los estudios concernientes a la formación del pensamiento histórico y la conciencia históricatemporal. Por último, queremos demostrar la importancia del desarrollo de la conciencia de la temporalidad en la educación para una ciudadanía crítica.

Palabras claves: Tiempo, tiempo histórico, didáctica de la historia, pensamiento histórico, conciencia histórica, educación para la ciudadanía.

This article reviews the relationship that has existed in the last 25 years between the researches on temporality learning and history teaching. The first works of evolutionary psychology were applied mechanically to history teaching. Their subsequent revisions carried to new proposals from the perspective of history education. Nowadays these perspectives are integrated in the studies on the formation of the historical thinking and the historical-temporal consciousness. Finally, we want to demonstrate the importance of developing the consciousness of temporality in critical citizenship education.
\end{abstract}

Key words: Time, historical time, history education, historical thinking, historical consciousness, citizenship education.

\section{Introducción}

Si repasamos la evolución de la didáctica de la historia en los últimos 25 años, podemos observar cómo han aumentado el número de investigaciones, revistas y propuestas de innovación en todos los países, hasta constituirse en un campo autónomo y, además, con una gran previsión de crecimiento, teniendo en cuenta la gran cantidad de preguntas que quedan por plantearse en la investigación y en la práctica educativa. Entre las problemáticas que más han evolucionado encontramos las relativas a cómo se adquiere o cómo se construye la temporalidad en los niños y niñas y jóvenes, y cómo las ideas del tiempo histórico influyen de manera decisiva en las concepciones respecto de la enseñanza y el aprendizaje de la historia (Santisteban 1999, 2005, 2006).

La evolución que intentamos describir en relación con el aprendizaje de la temporalidad comienza con unas primeras indagaciones desde la psicología piagetiana, para evolucionar hasta la perspectiva propia de la didáctica de la historia y las ciencias sociales, cuando esta disciplina se consolida con una investigación propia y con respuestas a las preguntas y problemas de la enseñanza y el aprendizaje del tiempo y de la historia. Este proceso implica una crítica a las investigaciones anteriores, así como a los trabajos basados, tan solo, en describir las ideas erróneas del alumnado en referencia a conceptos temporales. En este sentido, se ha dado un paso adelante hacia propuestas didácticas innovadoras para la enseñanza y el aprendizaje de la temporalidad histórica.

A grandes rasgos, desde la didáctica de la historia, la evolución de las ideas sobre la temporalidad van desde los estudios del aprendizaje del tiempo histórico y los conceptos temporales, como un aspecto esencial para la comprensión de la historia, hasta la temporalidad entendida como conciencia de ser humano, primero como memoria histórica y posteriormente como conciencia histórica (Rüsen 2007), es decir, donde nos descubrimos como seres temporales con la conciencia de la responsabilidad

* Universidad Autónoma de Barcelona. Barcelona, España. Correo electrónico: antoni.santisteban@uab.cat 
de que debemos conocer el pasado para poder comprender el presente y construir el futuro.

El trayecto que va desde los trabajos iniciales de los niveles de comprensión del tiempo histórico, hasta los estudios acerca del desarrollo de la conciencia histórica, también marca una preocupación cada vez mayor de cómo una determinada concepción de la temporalidad influye en la educación para la ciudadanía. De hecho, como defiende Freire (1978, 1979), la conciencia ciudadana crítica se forma a partir de una conciencia histórica-temporal. Comprender el tiempo histórico desde una perspectiva crítica y multicultural es una parte imprescindible para formarnos como personas y como ciudadanía democrática.

\section{Primeras investigaciones relativas al aprendizaje del tiempo histórico}

En la primera gran obra respecto de la noción de tiempo en la infancia Piaget (1978/1946) explica cómo los orígenes de su investigación están en la sugerencia que le hizo Albert Einstein, cuando se conocieron hace años en unos cursos internacionales de filosofía y psicología en la ciudad de Davos. Einstein sugirió a Piaget que investigara si la velocidad tenía un papel fundamental en la formación de la noción de tiempo, lo que le llevó finalmente a investigar también otros conceptos como duración, orden temporal o movimiento. Lo hizo con su método de análisis de las operaciones basado en el agrupamiento y aplicado con anterioridad a las ideas de los niños y niñas en los conceptos de número y cantidad, utilizando las mismas hipótesis.

Durante muchos años la obra de Piaget sirvió como referente también para las investigaciones de la enseñanza de la historia, de tal manera que se trasladaron las ideas relativas a los conceptos temporales estudiados por Piaget al aprendizaje de otros conceptos históricos. Así, lo que nació como un análisis de conceptos psicofísicos, acabó trasladándose a la didáctica de la historia, sin tener en cuenta otros factores cualitativos. Este es el caso de estudios como los de Peel (1967) o Hallam (1969), que mostraban las grandes dificultades del estudio de la historia, por la complejidad en la comprensión del tiempo histórico y que, por tanto, el aprendizaje de la historia no podía abordarse hasta una edad avanzada.

Otros estudios como los de Fraisse (1967), un discípulo de Piaget, que continuó sus investigaciones y elaboró el tratado más completo en la construcción del concepto tiempo, indagó en otros factores que influyen en la percepción del tiempo. El simple deseo de que el tiempo pase influye en nuestra experiencia, el aburrimiento, la espera o la prisa son factores decisivos para nuestra percepción del tiempo. Fraisse afirma que existe un "sentimiento de tiempo", en referencia a nuestra experiencia diaria del tiempo como vivencia.

Las aportaciones de Fraisse podrían haber cambiado algunas concepciones de cómo se comprende el tiempo y el tiempo histórico, pero su obra no tuvo demasiada repercusión.

El hecho que el tiempo pueda comprenderse a partir de las experiencias cotidianas y por el tipo de actividad que se realiza, es decir, que tuviera un componente importante de interpretación cualitativa, quiere decir que se podría haber avanzado en la comprensión de la historia como conciencia de la temporalidad humana, de narración histórica o de interpretación de las fuentes, más allá de la comprensión de determinados conceptos físicotemporales. Por ejemplo, no debemos confundir la cronología con la periodización, ya que mientras la cronología es objetiva y se aprende por memorización, la periodización requiere de la comprensión de la subjetividad del tiempo y de la arbitrariedad de las etapas históricas, según se priorice un cambio histórico u otro.

En el pasado un gran número de trabajos se destinaron a establecer la edad de adquisición de la cronología (Hannoun 1977; Zaccaria 1980) o las capacidades de seriación, pero muy pocas analizaron las relaciones temporales de causalidad o de intencionalidad (Dickinson y Lee 1978). Algunos investigadores reconocían que se estaba avanzando muy poco: "El confusionismo existente en torno al concepto de tiempo histórico ha sido muy grande" (Asensio, Carretero y Pozo 1989: 111). Por este motivo, otro de los investigadores pioneros en España consideraba que existía un vacío que dificultaba el aprendizaje de la historia, ya que la temporalidad todavía no formaba parte de los objetivos de la enseñanza de la historia y, como consecuencia: "...lo más frecuente es que la temporalidad se adquiera de forma espontánea e intuitiva, al margen de la escuela" (Pagès 1989: 107).

Autores como Shemilt $(1980,1987)$, en la evaluación del School Council History Project 13-16, llegaron a la conclusión de que con una metodología adecuada el alumnado puede llegar a adquirir 
importantes capacidades de pensamiento histórico y de comprensión de la temporalidad. Siguiendo estos argumentos, aparecen nuevas investigaciones que hacen hincapié en las capacidades de comprensión de la historia y la empatía (Ashby y Lee 1987; Lee, Dickinson y Ashby 1997).

\section{La importancia del lenguaje y la narración temporal en el aprendizaje de la historia}

Nuevos trabajos demostraron la importancia del lenguaje en la construcción de una narración temporal, de tal forma que cuando incorporamos diversos tipos de comunicación o materiales alternativos para estructurar los relatos de niños y niñas, se mejoran las nociones temporales e, incluso, se adelanta la edad de aprendizaje (Brown 1975; Stein y Glenn 1982). Autores como Booth (1987) o Calvani (1988) demostraron que eran necesarias más investigaciones acerca de la complejidad del proceso de comprensión de la historia.

Las revisiones de la investigación de Thornton y Vukelich (1988) o de Wineburg (1996) han corroborado que la temporalidad no solo depende de la madurez cognitiva, sino también de la experiencia o de la intervención educativa desde las primeras edades escolares. Thornton y Vukelich (1988) muestran cómo los niños y niñas de siete años dominan, por ejemplo, la ordenación temporal de las edades de los miembros de su familia.

Barton y Levstik (1996) investigaron respecto de las concepciones de la historia y del tiempo histórico de 58 niños y niñas de primaria. Utilizaron fotografías que había que ordenar temporalmente. Descubrieron que el alumnado de todas las edades tenían un sentido lógico de cómo ordenar de manera apropiada las imágenes, lo que indicaba que los niños y niñas pueden tener una capacidad significativa para entender la cronología histórica. Aunque los niños más pequeños confundían las fechas, los investigadores descubrieron que desarrollan importantes capacidades de comprensión de la historia previas -y hasta cierto punto independientes- al uso de fechas y otras formas de vocabulario más complejo.

Desde las primeras edades existen nociones temporales y capacidades para enfrentarse a una narración, lo que nos indica que hay muchas posibilidades para iniciar la enseñanza del tiempo histórico y la historia. Un ejemplo son los cuentos infantiles, que poseen una ordenación temporal lógica, así como componentes de temporalidad como el pasado o el futuro, la espera, etc. (Beer, 2003). Para algunos autores una de las claves ha sido simplificar las investigaciones y acercarlas a la vida cotidiana del alumnado (Bueno 1993).

La clave de las nuevas investigaciones estuvo en considerar a los niños y niñas desde las primeras edades capaces de hacer narraciones con estructuras temporales con una cierta coherencia, superando así las limitaciones que habían establecido investigaciones anteriores (Montangero 1984; Levin 1982; Richards 1982). Así, el lenguaje se convierte en un aspecto fundamental del aprendizaje de la historia (Santisteban y Pagès 2006; Pagès y Santisteban 2010; Aisenberg, 2012). Y la narración histórica se convierte en un instrumento esencial de la enseñanza y el aprendizaje del tiempo histórico y de la historia, en cualquier etapa educativa (Grau 2014; Llusà 2015). A partir de la narración histórica se desarrollan las diversas habilidades que forman el pensamiento histórico, desde la interpretación de las fuentes, la contextualización y la explicación causal e intencional. El relato histórico es la herramienta donde se construyen las interrelaciones entre pasado, presente y futuro, las tres categorías de la temporalidad humana.

\section{Los conceptos temporales se descubren como operadores cognitivos}

A partir del desarrollo del lenguaje construimos los diferentes conceptos temporales. El tiempo histórico no se define en sí mismo, sino como concepto de conceptos, que integra varios sistemas y subsistemas conceptuales. Las tipologías de los conceptos temporales son diversas, algunos conceptos son conocimientos específicos (cronología, década, periodización, ...), otras son instrumentos conceptuales que sirven para acceder a otros conocimientos históricos (revolución, ciclo, decadencia, ...). El aprendizaje de la historia requiere de una construcción progresiva de estructuras conceptuales temporales cada vez más complejas (Pagès y Santisteban 1999; Santisteban 1999; Santisteban 2005; Santisteban 2007).

Para Mattozzi (1988) el tiempo histórico es una macrocategoría analítica y un instrumento cognitivo:

A questo scopo dobbiamo pensare al tempo storico come a una macrocategoria analitica composta di molteplici categorie, che servono come strumenti cognitivi nella 
produzione delle informazioni, nella loro classificazione e nel loro ordinamento (Mattozzi 1988: 75).

Mattozzi (2002) considera que el pensamiento temporal está formado por una red de relaciones, donde se sitúan los hechos personales o históricos de una manera más o menos estructurada. Algunas propuestas como la de Audigier y Basuyau (1994) en Francia o la de Stow y Haydn (2000) en Inglaterra, piensan esta red de relaciones de manera algo diferente. Reivindican un lugar preeminente para la enseñanza de la cronología, pero relacionada con ciertos conceptos temporales básicos, como el cambio, la duración, la sucesión, los ritmos o las cualidades del tiempo histórico.

Algunos autores consideran que se deben cuestionar las categorías temporales que se presentan como categorías naturales, cuando no son más que construcciones sociales. También proponen deconstruir el concepto de periodización tal y como se utiliza (Segal 1991; Ferro 1991). Otros autores proponen deconstruir la idea de cronología occidental para construir nuevas cronologías (Milo 1991). Segal (1991) piensa que la periodización es un problema para la enseñanza de la historia. Considera que los períodos preconstruidos a menudo son un obstáculo para el desarrollo del pensamiento histórico. La periodización se impone a los manuales y los programas, pero no se enseña a periodizar.

Mattozzi considera que los conceptos temporales actúan como organizadores cognitivos, tanto en los acontecimientos de la vida cotidiana como en el proceso de comprensión del conocimiento histórico:

\section{L'infrastruttura degli organizzatori cognitivi è impercettibile ma essa è essenziale sia nel momento genetico della conoscenza storica sia nel memento in cui essa diventa conoscenza storiografica organizzata in testo. La capacità di operare con gli orga- nizzatori cognitivi è altrettanto essenziale nella comprensione della conoscenza storica comunicata (Mattozzi 1988: 69).}

El proceso de construcción de la estructura conceptual de la temporalidad histórica debe tener en cuenta los siguientes ámbitos: a) el cambio y la continuidad, como conceptos fundamentales que dan sentido al estudio de la historia, para explicar los procesos, por ejemplo, de transición, evolución o revolución, crisis, ciclos, modernidad, progreso o decadencia; b) las categorías de la temporalidad humana y las relaciones que se pueden establecer entre el pasado, el presente y el futuro, en el proceso de formación del pensamiento histórico y la conciencia histórica.

\section{La revisión del concepto de cambio (y continuidad)}

En nuestros días el concepto de cambio se asocia con las novedades tecnológicas de la era digital y con las posibilidades que ofrecen las redes para la información y la comunicación. Muchos autores, incluso, consideran que esta situación está afectando a nuestra vida transformando las relaciones personales. Los cambios en el trabajo o en la forma de viajar o de relacionarnos parece que están transformando nuestra percepción de la realidad social. Por otro lado, esta idea instalada en nuestras vidas de cambio continuo o de aceleración en la velocidad de los cambios provoca una sensación de incertidumbre, de riesgo o de miedo al futuro (Luhmann 2006; Burke 2007).

La idea de que vivimos en un mundo que cambia cada vez con mayor rapidez, nos sitúa en un estado de continua perplejidad o asombro, donde no podemos hacer nada por influir en el proceso, más allá de adaptarnos a las novedades. Lo que nos deja en una posición de indefensión ante lo que está por venir, que solo podemos esperar sin poder prepararnos para hacerle frente, ya que no sabemos qué será o cómo será. Nadie ha explicado esta realidad mejor que el sociólogo Bauman (2007) cuando califica los tiempos actuales de tiempos líquidos:

Resulta improbable que las formas, presentes o solo esbozadas, cuenten con el tiempo suficiente para solidificarse (...)... incluso más breve que el tiempo requerido para llevar a término un "proyecto de vida" individual (Bauman 2007: 7-8).

Podemos preguntarnos si esta situación afecta a cuestiones trascendentales de la vida de las personas o son aspectos superficiales. También podemos preguntarnos si es exclusiva del presente o ya se ha había dado en otras sociedades. Algunos personajes históricos han explicado sus experiencias en momentos que se vivían cambios 
de gran trascendencia. Estas vivencias nos sirven para entender el significado de la revolución del tiempo. Por ejemplo Mijail Gorbachov, en sus memorias referidas a su experiencia de gobierno en la antigua URSS explica:

"La elección interior en favor de los cambios no fue fácil para ninguno de nosotros. Todos éramos hijos de nuestra época. Todos estábamos poseídos por los dogmas ideológicos asimilados desde la infancia. Y la superación de esos dogmas fue un proceso complejo, diferente en cada caso y no siempre sincronizado. Unos recorrieron la senda y hasta el final. Otros se detuvieron a medio camino. Hubo quienes avanzaron unos pasos, se asustaron de las posibles consecuencias y comenzaron a retroceder. Todo esto repercutió en los ritmos, métodos y formas de movimiento hacia la perestroika" (Gorbachov 1994: 15).

El mismo personaje hace una reflexión de su época histórica y considera que se están viviendo cambios revolucionarios y la llegada de una nueva civilización:

La humanidad se halla en un momento de cambio brusco. El mundo está en el umbral de mutaciones radicales. No se trata de un paso más de una etapa a otra, como ha vivido tantos la historia universal. Muchos indicios confirman que se trata de un cambio de envergadura y que toca directamente el sentido de la historia, de la llegada de una nueva civilización (Gorbachov 1994: 24).

Si retrocedemos algo más en el tiempo podemos situarnos entre los siglos XVIII y XIX para comprender lo que escribe Chateaubriand (17681848) en sus Memorias de ultratumba, que son una especie de biografía en la que repasa sus vivencias como intelectual y político:

“Toda la geografía ha cambiado... Si comparo dos globos terráqueos, uno del comienzo y otro del final de mi vida, no los reconoceré. Se ha descubierto y poblado una cuarta parte de la Tierra... Bonapartismo, orleanismo, legitimismo, son tres cosas muertas. Pertenecen a la monarquía y a la aristocracia, que están muertas. El partido democrático es el único que progresa porque marcha hacia el futuro... El futuro es del pueblo" (Chateaubriand, citado en Coma y Santisteban 1997: 299).

Podemos pensar que las anteriores personas han vivido épocas exclusivas de la edad contemporánea donde se dieron cambios radicales, que les hicieron percibir el tiempo acelerado, la sociedad en transformación continua, donde no hay permanencia, solo cambio e incertidumbre. Nada más lejos de la realidad, la historia del mundo está llena de este tipo de momentos históricos, tal vez tan solo es diferente la forma de explicarlos. A continuación reproducimos una reflexión atinente al cambio y la continuidad dentro del "Poema de Gilgamesh", escrito hace más de cuatro mil seiscientos años en Sumeria:

No hay permanencia. ¿Construimos una casa para que dure siempre?, ¿sellamos un pacto para mantenerlo todo el tiempo?, ¿se reparten los hermanos una herencia para conservarla siempre?, ¿soportará la época de la inundación de los ríos?... Desde los días de antaño no hay permanencia (Sanders, 1960: The Epic of Gilgamesh, 104, citado en Whitrow 1990: 48).

Las personas, las sociedades o las culturas cambian, pero siempre existen elementos de continuidad que nos permiten enlazar y relacionar los hechos, los personajes y las interpretaciones. El binomio cambio-continuidad incluye a la vez otros conceptos, que a veces se utilizan como sinónimos de cambio, pero que en realidad lo matizan o lo complementan, como son evolución y revolución, crecimiento y desarrollo, transición y transformación, progreso, modernidad y decadencia.

El cambio es el concepto más importante del estudio de la historia. El análisis del cambio hace posible la construcción de una racionalidad en el estudio del pasado, que no sería posible sin una diferenciación de las sociedades en el tiempo. Sin cambio no habría tiempo. El cambio social marca la periodización histórica y a su alrededor giran la interpretación, la explicación histórica, la causalidad y la intencionalidad de los argumentos. La mayoría de ocasiones la validez historiográfica del análisis 
de los hechos históricos, radica en el tratamiento que reciben los cambios sociales, cómo se explican y cómo se interpretan.

El estudio de los cambios históricos del pasado, en especial aquellos que significaron avances en las sociedades, como la consecución de mejores condiciones de vida o de trabajo, o los derechos políticos o sociales, deben ayudarnos a comprender que se pueden conseguir cambios sociales en nuestro presente y que el futuro está por decidir. La percepción del cambio como algo inevitable o la no permanencia de elementos de nuestro pasado, produce pasividad y falta de participación, en el sentido contrario de una educación para la ciudadanía democrática y crítica.

\section{El concepto de futuro irrumpe en la enseñanza de la historia}

El interés por el futuro en la historia surge del análisis del pasado y de la interrelación que guarda con el presente de las personas y con sus proyecciones: "Como historiador me preocupa siempre el futuro: ya sea el futuro tal como ya ha nacido de algún pasado anterior, o tal como es probable que nazca del continuo del pasado y el presente" (Hobsbawm, 1998: 118). El concepto de futuro es inherente al estudio de la historia y afecta inevitablemente a la tarea del historiador (Pomian 2007). Es en realidad la meta más importante del estudio de la historia (Carr 2003).

Incluso la interpretación del pasado puede cambiar según la evolución de nuestra percepción del futuro (Cruz 2002; Lowenthal 1998; Burke 2007). Esta es una de las grandes metas de la enseñanza de la historia: "en cada época y para cada generación, se abren diversos futuros, por lo tanto, también en nuestro tiempo tenemos ante nosotros diferentes futuros sobre los que debemos decidir" (Santisteban y Anguera 2014).

El historiador Christopher Clark (2014) ha analizado la Gran Guerra de 1914, para concluir que la guerra se podía haber evitado (20 millones de muertos y 21 millones de heridos), incluso considera que era "improbable" que ocurriera. Clark (2014) presenta a los protagonistas y los hechos de tal forma que se podrían distinguir "las semillas de otros futuros tal vez menos terribles" (30). Este trabajo nos muestra que el pasado puede cambiar a partir de nuestras ideas concernientes al presente o el futuro: "Los cambios en nuestro mundo han alterado nuestra perspectiva de los sucesos de 1914" (Clark, 2014, 25).

La historia reclama la idea de futuro como esperanza de posibles cambios sociales (Anderson, 1996; Gadamer, 1993; Todorov, 2000). Las relaciones entre el pasado, el presente y el futuro se han convertido en el eje central de la reflexión histórica acerca del tiempo:

"Lo que es más, la mayor parte de la acción consciente de los seres humanos que se basa en el aprendizaje, la memoria y la experiencia constituye un inmenso mecanismo que sirve para afrontar constantemente el pasado, el presente y el futuro. Intentar prever el futuro interpretando el pasado es algo que las personas no pueden evitar. Tienen que hacerlo" (Hobsbawm 1998, 53)

Ferro (1999) propone que el estudio de la historia sustituya la historia-discurso por la historiaproblema, que se establezcan interacciones espaciales y temporales, de la micro a la macrohistoria. Así, la relación entre pasado, presente y futuro se convierte en el aspecto central del estudio de la historia, ya que amplía nuestra perspectiva de los hechos y de los cambios históricos, de la interpretación de la experiencia y de las posibilidades de la prospectiva.

Otros autores como Evans (1996) o Audigier (2003) proponen una educación crítica en valores democráticos, basada en estas relaciones entre pasado, presente y futuro, como centro de la enseñanza de la temporalidad y de la historia, pero también son la última finalidad de la formación ciudadana como parte irrenunciable de su cultura democrática. Para Dewey (1995) el punto de partida debe de ser siempre una situación problemática del presente. $\mathrm{La}$ enseñanza de la historia y de las ciencias sociales deben partir de problemas sociales relevantes que nos preocupan, analizar su evolución histórica, hasta llegar a construir una proyección hacia el futuro.

Slaughter (2000) nos propone repensar las relaciones entre las tres categorías temporales, a partir de tres tipos de imágenes. Una imagen con relaciones unidireccionales, es decir, el pasado afecta al presente y este al futuro. En la segunda, las relaciones son abiertas y los flujos no van solo en una dirección, sino que pasado y futuro se influyen continuamente. Finalmente, nos propone una figura en forma de tejido que representa al presente, confeccionado con los hilos del pasado 
y del futuro. En esta última figura el pasado y el futuro son reales y perdurables, y el presente es solo el "aquí y el ahora".

Inayatullah (1998) propone acabar con una larga tradición de futuristas que ignoraban la importancia del conocimiento del pasado. Es necesario comprender la macrohistoria, para analizar las causas y los mecanismos del cambio histórico, lo que nos permite ofrecer estructuras útiles para interpretar el futuro. Para este autor es esencial tener en cuenta las aportaciones que puede hacer la historia a la prospectiva. También Bussey et al. (2012) defiende las aportaciones de la historia al estudio del futuro, ya que el análisis del pasado nos muestra procesos, opciones y alternativas.

Para Oyserman y James (2011) proponen también un cierto cambio en las ideas respecto del futuro en la enseñanza. Consideran que nuestras identidades se construyen con la imagen actual que tenemos de nosotros mismos, pero también a partir de la imagen del futuro que pensamos. Argumentan que el futuro tiene una gran influencia en nuestras ideas relativas a la participación en la sociedad. Pero proponen no presentar el futuro en la enseñanza como algo que esperamos que "llegue". Debemos enseñar que el futuro empieza "ahora".

\section{Las relaciones entre la formación del pensamiento histórico y el desarrollo de la conciencia histórica-temporal}

En nuestras investigaciones (1) hemos analizado lo que significa pensar históricamente hasta definir tres tipos de competencias históricas: a) la interpretación de las fuentes históricas primarias y secundarias, y la solución de preguntas-problemas históricos; b) las relacionadas con la imaginación histórica (empatía, contextualización y juicio moral); c) la narración como forma esencial del discurso histórico, que puede evolucionar como explicación histórica causal o intencional (Santisteban, González y Pagès, 2010; Santisteban, 2010). En la Figura 1

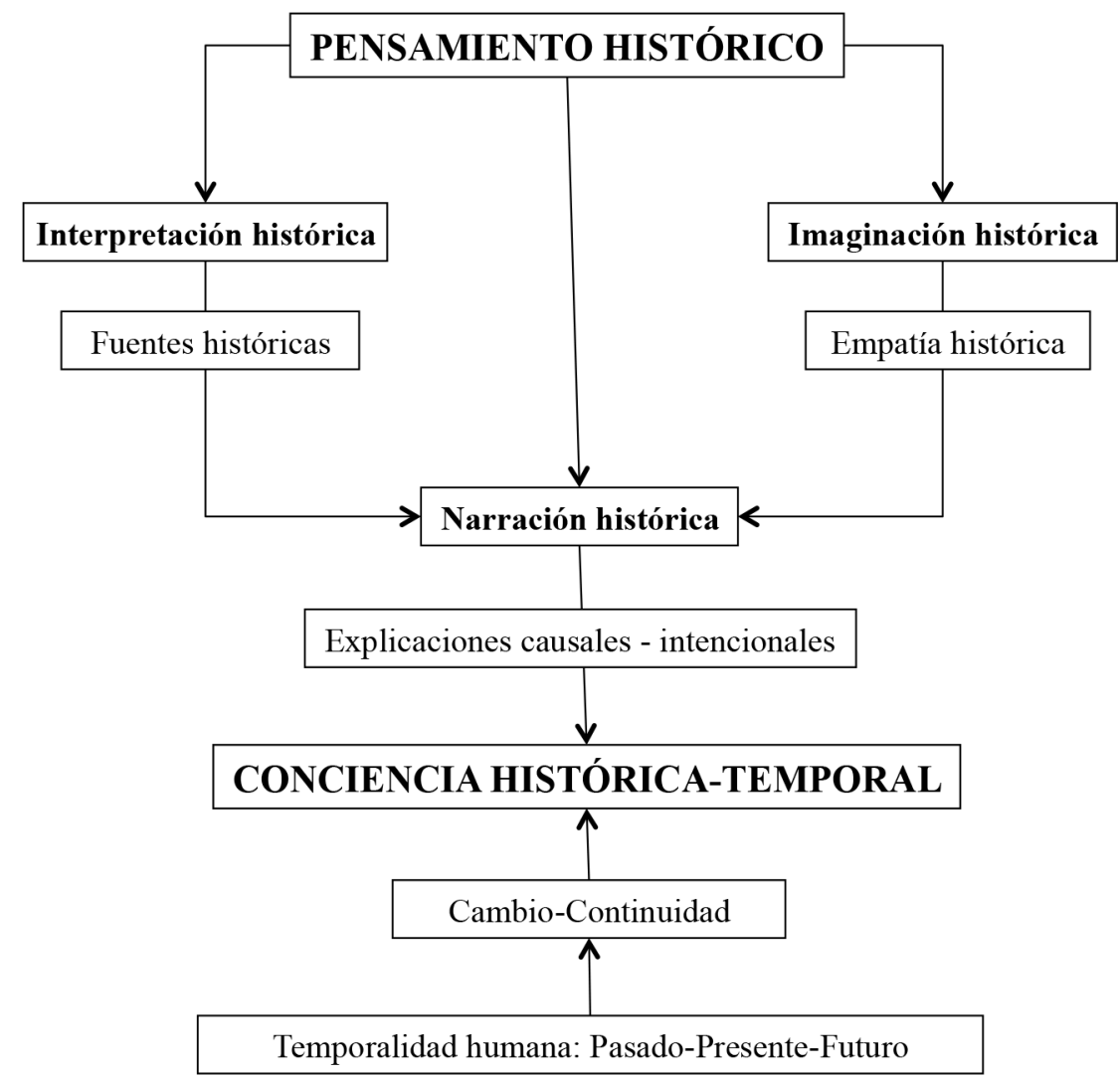

Figura 1. Esquema de desarrollo del pensamiento histórico. 
sintetizamos nuestras ideas referidas a las relaciones entre la formación del pensamiento histórico y la conciencia histórica-temporal.

La formación del pensamiento histórico tiene como objetivo principal el desarrollo de la conciencia histórico-temporal, para la comprensión de los cambios y continuidades en la historia, y para comprender las relaciones entre pasado, presente y futuro (Rüsen, 2001). Expresado de otra forma, podemos afirmar que en la formación de la conciencia histórica-temporal se sintetizan todas las competencias del pensamiento histórico (Seixas, 2004; Rüsen, 2004). Su desarrollo es paralelo al de las habilidades necesarias para la interpretación, la imaginación y la narración histórica.

Por otro lado, hemos de distinguir entre memoria histórica y conciencia histórica, aunque sean conceptos de la misma naturaleza (Laville, 2003). Desde la enseñanza de la historia son conceptos diferentes. La memoria histórica es reivindicación del pasado. La conciencia histórica utiliza procedimientos para la construcción de la temporalidad y del cambio-continuidad, en un proceso que va del pasado al futuro. Para Rüsen (2007), la memoria se dirige al pasado y la conciencia histórica rescata el pasado para analizar cómo pensar el futuro. La memoria se relaciona con la imaginación y la empatía histórica. La conciencia histórica está más relacionada con las capacidades de interpretación y de narración histórica (Rüsen 2001, 2007). La memoria es el ejemplo o la lección moral, mientras que la conciencia histórica es el análisis de las posibilidades, la evaluación de los cambios sociales, de las rupturas y continuidades (Pagès y Santisteban, 2008).

El estudio más amplio realizado respecto de la conciencia histórica de los jóvenes es el que dirigieron Angvik y Von Borries (1997), a partir de una encuesta a jóvenes de 15 años en 25 países de Europa, además de Israel, Palestina y Turquía. Sus conclusiones indican que la formación de la conciencia histórica de los chicos y chicas de esta edad, depende de las relaciones complejas que establecen entre la interpretación del pasado, la percepción del presente y las esperanzas dirigidas al futuro. La investigación parece demostrar que la enseñanza de la historia juega un papel poco trascendente en la formación de la conciencia histórica y los valores democráticos. Los resultados publicados, por ejemplo del alumnado de Francia, ofrecen conclusiones parecidas. Según sus respuestas, la conciencia histórica de los jóvenes es muy débil (Tutiaux- Guillon y Mousseau 1998).

Wineburg (2000) en Estados Unidos indagó acerca de la formación del sentido histórico en los estudiantes de secundaria. Este investigador entrevista a estudiantes y sus familias en diferentes ocasiones en el curso de dos años y medio. Descubre cómo las visiones de la historia de los estudiantes están ancladas en el discurso familiar, y este es apoyado a veces por la escuela y otras veces por los medios de comunicación o la experiencia personal. Pero, en todo caso, la enseñanza de la historia no parece jugar el papel que debería en la formación del pensamiento histórico para llegar a construir una conciencia histórica en la ciudadanía.

\section{Investigaciones del grupo GREDICS sobre la conciencia histórica}

En diferentes investigaciones hemos analizado el nivel de desarrollo de la conciencia histórica del alumnado de educación secundaria. También hemos experimentado materiales alternativos para intentar modificar su conciencia de temporalidad. En una de estas investigaciones aplicamos una unidad didáctica con chicos y chicas de 15 años de dos centros de educación secundaria de Barcelona, uno público y otro privado. La temática tratada fueron las migraciones a lo largo de las diferentes épocas históricas, a partir de 6 sesiones de trabajo. La última actividad solicitaba al alumnado que imaginara una situación ficticia del futuro, dentro de 50 años, en la que su hija de 25 años habría emigrado a un país del sur de África para buscar trabajo, ya que la situación de Europa había empeorado mucho. La hija les pedía consejo relacionado con los problemas que estaba viviendo como inmigrante en un país extraño, donde no conocía la lengua ni las costumbres, mediante un correo electrónico que debían contestar (González-Monfort, Henríquez, Pagès, Santisteban 2010).

Los resultados de la investigación, analizando la última actividad descrita, muestran las muchas dificultades que tiene el alumnado de estas edades para situarse en el tiempo y para relacionar pasado, presente y futuro, es decir, para construir su conciencia histórica. El alumnado ofrece consejos muy ingenuos en sus escritos sin tener en cuenta lo que había estudiado pertinente a situaciones problemáticas relacionadas con la emigración anteriormente. Ante la sorpresa de los y las investigadoras solo una 
pequeña minoría del alumnado utiliza el conocimiento del pasado para reflexionar de la situación planteada. Son muy pocos los textos narrativos que relacionan pasado-presente-futuro.

En otra de nuestras investigaciones experimentamos con el alumnado de educación secundaria una unidad didáctica acerca de "Límites, fronteras y muros" (Santisteban, 2012). Se realizaron también 6 sesiones de trabajo, con una parte relativa a la perspectiva histórica de la existencia de muros en el mundo, desde el Muro de Adriano o la Muralla China, hasta el Muro de Berlín, Belfast, Corea, etc. La última actividad planteaba una situación ficticia en la que el gobierno español había decidido levantar un gran muro de hormigón en la frontera con Marruecos. Después de un debate y el desarrollo de cuestiones respecto de la argumentación y contraargumentación, finalmente, el alumnado debía decidir cuál sería su posición si tuvieran el poder político para tomar decisiones.

En la investigación participaron 234 alumnos de $2^{\circ}, 3^{\circ}, 4^{\circ}$ de ESO y $1^{\circ}$ de Bachillerato (13$16 / 17$ años), de diversos centros de la periferia de Barcelona. Los resultados de esta investigación mostraron, como en la anterior, que los conocimientos históricos no eran utilizados en la argumentación del alumnado para tomar decisiones de la situación problemática en esta frontera, en la que cada día cientos de personas intentan traspasarla para llegar a España y a Europa.

La influencia de la cultura familiar y de los medios de comunicación predomina por encima del debate escolar. Solo unos pocos alumnos y alumnas cambian de opinión respecto de la posición manifestada al principio de la unidad didáctica en relación con la necesidad o no de crear muros en Europa. Pero como aspecto destacable de cambio, aumenta la empatía respecto de las personas que están al otro lado del muro. Y como otro aspecto positivo, un porcentaje significativo de alumnado manifiesta la esperanza de que en el futuro no haya la necesidad de construir más muros.

En la investigación en la que estamos trabajando en este momento nos hemos centrado en el proceso de literacidad crítica del alumnado, en relación con la información que están ofreciendo los medios de comunicación relacionado con la problemática de los refugiados y refugiadas en Europa (Santisteban et. Al 2016). Se trata de analizar si el alumnado distingue entre hechos y opiniones, si es capaz de argumentar referente a la veracidad o la fiabilidad de una información, si identifica la ideología de una fuente o sus silencios. En todo caso la literacidad crítica se dirige a un objetivo final irrenunciable que es la acción social responsable.

Hasta el momento la investigación se ha aplicado a cuatro centros de educación secundaria de Barcelona y comarcas cercanas en los cursos de $3^{\circ}$ y $4^{\circ}$ (15-16 años), con un cuestionario de dos sesiones, aunque la investigación se sigue ampliando. Uno de los aspectos destacados de los resultados obtenidos hasta el momento es que las situaciones históricas que el alumnado conoce, por ejemplo la de los exiliados y refugiados españoles después de la guerra civil -recordadas en un video al inicio de la unidad-, no son comparadas, utilizadas o recordadas de ninguna manera en sus argumentaciones en la última parte del cuestionario, donde se solicita que aporten soluciones, alternativas, etc. En este caso, parecía obvio que el pasado nos mostraba una lección para el futuro, pero el conocimiento histórico se descarta como herramienta de argumentación o como saber útil al pensamiento crítico.

En estos ejemplos de investigaciones hemos querido demostrar las dificultades que el alumnado tiene para formar su conciencia histórica y relacionar pasado, presente y futuro. Pero también debe quedar clara la importancia de esta necesidad en la enseñanza y hemos señalado algunas posibilidades de investigación, pero también de innovación, por medio de la perspectiva histórica de temáticas problemáticas de la actualidad.

\section{Conclusiones}

En este texto hemos hecho un recorrido desde la enseñanza del tiempo histórico hasta la construcción en la escuela de la temporalidad humana como conciencia histórica. Esta ha sido la gran revolución en la enseñanza de la historia de fines del siglo XX y el gran reto que tenemos el profesorado de historia para el siglo XXI, es decir, descubrirnos como seres humanos en el tiempo (Levstik 2014). Como afirma Gadamer (1993): "La aparición de una toma de conciencia histórica es verdaderamente la revolución más importante de las que hemos experimentado tras la llegada de la época moderna" (41).

La conciencia histórica forma parte de la conciencia ciudadana, ya que la historia es una aportación esencial a la educación democrática. La conciencia histórica es conciencia crítica, como afirma Freire $(1978,1979)$, para quien la conciencia 
de la temporalidad es parte de una conciencia social y una cultura política. La conciencia histórica nos ayuda a tomar decisiones y la historia se convierte en un instrumento de liberación y de intervención social, como parte esencial de la educación democrática.

Tal vez para una parte importante del profesorado puede parecer que el estudio del pasado es suficiente para comprender las claves del presente, pero nuestras investigaciones muestran con claridad que estas relaciones no son nada fáciles y deben trabajarse con detenimiento. Por otro lado, la prospectiva implica capacidades de orden superior que requieren de la formación del pensamiento histórico, de la interpretación, la imaginación histórica y la narración, para construir una conciencia que relacione pasado, presente y futuro ante preguntas o problemas históricos reales. El alumnado movilizará sus conocimientos acerca del pasado si estos se conectan con claridad con sus imágenes del futuro. Y este proceso es también responsabilidad del profesorado de historia, que debe conocer los resultados de las investigaciones cuando las mismas pretenden analizar los problemas de la práctica y, como consecuencia, aportan resultados para la mejora de la enseñanza de la historia.

Algunos autores insisten en que vivimos en unos tiempos líquidos, de incertidumbre o de riesgo, lo que hace de la enseñanza de la historia algo inestable. Pero nosotros proponemos deconstruir el tiempo histórico en la escuela, relacionar las tres categorías de la temporalidad humana y revisar el futuro en función de un nuevo pasado reescrito por las próximas generaciones. Y formar la conciencia histórica-temporal como una nueva manera de observarnos a nosotros mismos, hombres y mujeres, como seres humanos en la historia.

\section{Referencias Citadas}

Aisenberg, B.

2012 Usos de la escritura en la enseñanza de la historia. Clío \& Asociados, 16, 99-105.

Anderson, $\mathrm{P}$.

1996 Los fines de la historia. Barcelona: Anagrama.

Angvik, M; Von Borries, B. (eds.)

1997 Youth and History. A comparative European Survey on Historical Consciousness and Political Attitudes among Adolescents. Hambourg: Körber-Stiftung.

Asensio, M.; Carretero, M.; Pozo, J.I.

1989 La comprensión del tiempo histórico. Carretero, M.; Pozo, J.I.; Asensio, M. (comp.). La enseñanza de las Ciencias Sociales (pp. 103-137). Madrid: Aprendizaje Visor.

Ashby, R.; Lee, P.

1987 Children's concepts of empathy and unders tanding in history. Portal, C. (ed.). The history curriculum for teachers (pp. 62-68). Londres: Palmer Press.

Audigier, F.

2003 "Histoire scolaire, formation citoyenne et recherches didactiques". Baques, M-C.; Bruter, A.; Tutiaux-Guillon, N. (eds.). Pistes didactiques et chemins d'historiens. Extes offerts à Henri Moniot. Paris: L'Harmattan. 241-263.

Audigier, F.; Basuyau, C.

1994 "La chronologie n'est pas l'histoire, et pourtant...". IREHG, 1. Revue des Instituts de recherche pour l'enseignement de l'Histoire, Géographie et Groupes Associés, 79-90.

Barton, K.C.; Levstik, L.S.

1996 "'Back when God was around and everything': Elementary children's understanding of historical time". American Educational Research Journal, 33. 419-454.

Bauman, Z.

2007 Los retos de la educación en la modernidad líquida. Barcelona: Gedisa.

Beer, G.

2003 La hora de los cuentos y sus futuros. Ridderbos, K. (ed.). El tiempo. Madrid: Cambridge University Press. 115-129.
Booth, M.B.

1987 Ages and concepts: A critique of the Piagetian approach to history teaching. Portal, C. (ed.). The history curriculum for teachers. Londres: Palmer Press. 22-38.

Brown,A.L.

1975 Recognition, Reconstruction and Recall of Narrative Sequences by Preoperational Children. Child Development, 46, 156-166.

Bueno, M.B.

1993 El desarrollo del conocimiento humano sobre el tiempo. Infancia y Aprendizaje, 61, 29-54.

Burke, P.

2007 Historia y teoría social. Buenos Aires: Amorrortu.

Bussey, M. et al.

2010) Framing adaptive capacity through a history-futures lens: Lessons from the South East Queensland Climate Adaptation Research Initiative. Futures, nº44, pp.385-397.

Calvani, A.

1988 Il bambino, il tempo, la storia. Firenze: La Nuova Italia.

Carr, E.H.

2003 ¿Qué es la historia? Barcelona: Ariel (nueva edición completa).

Clark, C.

2014 Sonámbulos. Cómo Europa fue a la guerra en 1914. Barcelona: Galaxia Gutenberg.

Coma, J.; Santisteban, A.

1997 La crisis del Antiguo Régimen y las Revoluciones liberales burguesas, en Benejam, P.; Pagès, J. (coord.): Ciencias Sociales. Contenidos, Actividades y Recursos. Guías Praxis para el Profesorado de ESO (pp. 239-302). Barcelona: Praxis.

Cruz, M. (ed.)

2002 Hacia dónde va el pasado. El porvenir de la memoria en el mundo contemporáneo. Barcelona: Paidós.

Dewey, J.

1995 Democràcia i escola. Barcelona: Eumo. 
Dickinson, A.K.; Lee, P.J.

1978 History Teaching and Historical Understanding. London: Heinemenn.

Evans, R. W.

1996 "A critical approach to tecahing United States History". Evans, R.W./Saxe, D.W. (ed.). Handbook On Teaching Social Issues. Washington: National Council for the Social Studies (NCSS). 152-160.

Ferro, M.

1991 "Visions de l'histoire et périodisation, une typologie". AA.VV. Périodes. La construction du temps historique. Actes du Ve Colloque d'histoire au present. Éditions de l'École des Hautes Études en Sciences Sociales et Histoire au présent. Paris. 99-101.

Ferro, M.

1999 Las fuentes de la conciencia histórica. ¿Crisis de la historia o de la disciplina? Signos Históricos, I.2 (diciembre), pp. 139-152.

Fraisse, P.

1967 Psychologie du temps. París: PUF (2a ed. corregida i augmentada).

Freire, $\mathrm{P}$.

1978 La educación como práctica de la libertad. Madrid: Siglo XXI.

Freire, $\mathrm{P}$.

1979 Pedagogía del oprimido. Madrid: Siglo XXI.

Gadamer, H-G.

1993 El problema de la conciencia histórica. Madrid: Tecnos.

González-Monfort, N., Henríquez, R., Pagès, J.; Santisteban, A. 2010 Empatía histórica para un presente cambiante. La enseñanza y el aprendizaje de la comprensión histórica a través de una experiencia didáctica. Pagès, J. y Gónzalez-Monfort, N. (coords.). La construcció de les identitats i l'ensenyament de les Ciències Socials, de la Geografia i de la Història (pp. 145-157). Barcelona: Servei de Publicacions UAB.

Gorbachov, M.

1994 Memorias de los años decisivos (1985-1992). Madrid: Globus.

Grau, F.

2014 Els relats històrics en les aules de secundària. Un cas d'investigació-acció. Tesis Doctoral. Universitat Autònoma de Barcelona.

Hallam, R.

1969 "Piaget and the Teaching of History", a Educational Research, 12, 1, 3-12. Trad. cast. en Coll, C. (comp.). Psicología genética y aprendizajes escolares. Madrid: Siglo XXI, 1983, 167-181.

Hannoun, $\mathrm{H}$.

1977 El niño conquista el medio. Buenos Aires: Kapelusz.

Hobsbawm, E.

1998 Sobre la historia. Barcelona: Crítica.

Inayatullah, $\mathrm{S}$.

1998). Macrohistory and futures studies. Futures, $\mathrm{N}^{\circ} 30,5$, 381-394.

Laville, C.

2003 Pour une education historique critique, qu' attendre du courant de la conscience historique?, en Tutiaux-Guillon, $\mathrm{N}$. (Coord.). Identités, mémoires, conscience historique, Saint Étienne: Publications de l'Université Sint Éttienne.

Lee, P.; Dickinson, A.; Ashby, R.

1997 'Just another emperor': Understanding action in the past. International Journal of Educational Research, 27, 3. 233-244.
Levin, I.

1982 The Nature and Development of Time Concepts in Children: the Effects of Interfering Cues. Friedman,W.J. (ed.). The Developmental Psichology of Time. Nova York: Academic Press. 47-85.

Levstik, L.

2014 What Can History And The Social Sciences Contribute To Civic Education?, in Pagès, P.; Santisteban, A. (ed.): Una mirada al pasado y un proyecto de futuro. Investigación e innovación en la didáctica de las ciencias sociales (pp. 43-51). Barcelona: Servei de Publicacions de la UAB/ AUPDCS.

Llusà, J.

2015 Ensenyar història des de la contemporaneïtat. Estudi de cas sobre la construcció i aplicació del concepte contemporaneïtat a l'ESO. Tesis Doctoral. Universitat Autònoma de Barcelona.

Lowenthal, D. 1998 El pasado es un país extraño. Madrid: Akal.

Mattozzi, I.

1988 I bambini, il tempo, la storia: educazione temporale e curricolo di storia nella scuola elementare. C.I.D.I. di Firenze (a cura di). Tempo e spazio, dimensioni del sapere. Dalle ipotesi teoriche alle practiche didattiche. Milano: Mondadori. 65-81.

Mattozzi, I.

2002 Presentazione per il docente. La formazione del pensiero temporale negli adolescenti. Perillo, E. (a cura di). $L a$ storia. Istruzione per l'uso. Materiali per la formazione de competenze temporali degli studenti. Napoli: Tecnodid. 9-22.

Milo, D.

1991 Trahir le temps. Paris: Les Belles Lettres.

Montagero, J.

1984 Perspectives actualles sur la psychogenese du temps. L'Anné Psichologique, 84, 433-460.

Oyserman, D.; James, L.

2011 Possible Identities, in Schwartz, S.J.; Luyckx, K.; Vignoles, V. (ed.): Handbook of Identity Theory and Research, Vol.1, Structures and Processes, Nueva York: Springer, pp. 117-145.

Pagès, J.; Santisteban, A.

1999 La enseñanza del tiempo histórico: una propuesta para superar viejos problemas. AAVV: Un currículum de Ciencias Sociales para el siglo XXI. Qué contenidos y para qué (pp. 187-207). Sevilla: Díada.

Pagès, J.; Santisteban, A.

2008 Cambios y continuidades: Aprender la Temporalidad Histórica. Jara, M. A. (comp.): Enseñanza de la historia. Debates y propuestas (pp. 91-124). Río Negro: Educo.

Pagès, J.; Santisteban, A.

2010 La enseñanza y el aprendizaje del tiempo histórico en la educación primaria. Cad. CEDES vol. 30, 82, 278-309.

Peel, E.A.

1967 Some problems in the psychology of history teaching. Burston, W.H.; Thompson, D. (eds.). Studies in the nature and teaching of history. Londres: Roudledge et Kegan Paul. 159-190.

Piaget, J.

1978 El desarrollo de la noción de tiempo en el niño. México: Fondo de Cultura Económico (Primera edición de 1946). 
Pomian, K.

1988 Temporalidad histórica/tiempo, a Le Goff, J.; Chartier, R. i Revel, J. (dir.) La nueva historia (pp. 590-592). Bilbao: Mensajero.

Richards, D.D.

1982 Children's time concepts: Going the distance. Friedman,W.J. (ed.). The Developmental Psichology of Time. Nova York: Academic Press.

Rüsen, J.

2001 What is Historical Consciousness? A Theoretical Approach to Empirical Evidence, Paper presented at Canadian Historical Consciousness in an International Context: Theoretical Frameworks, University of British Columbia, Vancouver, BC

Rüsen, J.

2004 Historical Consciousness: Narrative Structure, Moral Function, and Ontogenetic Development. Seixas, P. (ed.): Theorizing Historical Consciousness, Toronto: University of Toronto Press, pp. 63-85.

Rüsen, J.

2007 Memory, history and the quest for the future. Cajani, L. (ed.): History Teaching, Identities and Citizenship. European Issues in Children's Identity and Citizenship, 7, CiCe. Stoke on Trent. Trentham Books, 13-34.

Santisteban, A.

1999 Aprender el tiempo histórico: deconstruir para reconstruir. Historiar, 1: 141-150.

Santisteban, A.

2005 Les representacions i l'ensenyament del temps històric. Estudis de cas en formació inicial de mestres de primària en Didàctica de les Ciències Socials. Universitat Autònoma de Barcelona. Tesis Doctoral.

Santisteban, A.

2006 Une proposition conceptuelle pour la recherche et l'enseignement du temps historique. Le Cartable de Clio (GDH, Éditions LEP, Lausanne), Nº 6, pp. 77-90.

Santisteban, A.

2007 Una investigación sobre cómo se aprende a enseñar el tiempo histórico. Enseñanza de las Ciencias Sociales, 6: 19-29.

Santisteban, A.

2010 La formación en competencias de pensamiento histórico, en Clío \& Asociados. La Historia Enseñada, 14, 34-56.

Santisteban, A.

2012 La investigación sobre el desarrollo de la competencia social y ciudadana para una participación crítica. De Alba, N.; García, F.; Santisteban, A. (eds.). Educar para la participación ciudadana en la enseñanza de las ciencias sociales (pp. 277-286). Sevilla: Díada. Vol. II.

Santisteban, A.; Anguera, C.

2014 Formación de la conciencia histórica y educación para el futuro. Clío \& Asociados, 18-19, 249-267.

Santisteban, A.; González-Monfort, N.; Pagès, J.

2010 Una investigación sobre la formación del pensamiento histórico. Ávila, R.M., Rivero, P.; Domínguez, P.L. (coords.): Metodología de investigación en Didáctica de las Ciencias Sociales (pp.115-128)., Zaragoza: Institución "Fernando el Católico" (C.S.I.C)/ AUPDCS.

Santisteban, A. ; Pagès, J.

2006 La enseñanza de la historia en la educación primaria. Casas, M. y Tomàs, C. (coord.) Educación Primaria. Orientaciones y Recursos, 468/129-468/183. Barcelona: Wolters Kluwer Educación.
Santisteban, A.; Tosar, B; Izquierdo, A.; Llusà, J.; Canals, R.; González, N.; Pagès, J.

2016 La literacidad crítica de la información sobre los refugiados y refugiadas: construyendo la ciudadanía global desde la enseñanza de las ciencias sociales. García Ruiz, C.R.; Arroyo, A.; Andreu, B. (eds.), Deconstruir la alteridad desde la didáctica de las ciencias sociales: educar para una ciudadanía global (pp. 550-560). Las Palmas de Gran Canaria: Universidad de Las Palmas/AUPDCS.

Segal, A.

1991 Didactique de la périodisation: le 'moyen âge' comme obstacle à l'intelligence des origines de l'Occident?. AAVV. Périodes. La construction du temps historique. Actes du Ve Colloque d'histoire au present (pp. 105-114).. Paris: Éditions de l'École des Hautes Études en Sciences Sociales et Histoire au présent.

Seixas, P. (ed.)

2004 Theorizing Historical Consciousness. Toronto: University of Toronto Press.

Shemilt, D.J.

1980 History 13-16: Evaluation study. Edimbourg: Holmes McDougall.

Shemilt, D.J.

1987 El proyecto 'Historia 13-16' del Schools Council: pasado, presente y futuro. MEC. La Geografía y la Historia dentro de las Ciencias Sociales: hacia un currículum integrado (pp. 173-207). Madrid: MEC.

Slaughter, R.

2000 Futures concepts and powerful ideas. Melbourne: Futures StudyCentre.

Stein, N.L.; Glenn, C.G.

1982 Children's Concept of Time: The Development of a Story Schema. Friedman,W.J. (ed.). The Developmental Psichology of Time. Nova York: Academic Press. 255-282.

Stow, W.; Haydn, T.

2000 Issues in the teaching of chronology. Arthur, J.; Phillips, R. (eds.). Issues in History Teaching. London: Routledge. 83-97.

Thornton,S.J.; Vukelich,R.

1988 Effects of Children's Understanding of Time Concepts on Historical Understanding. Theory and Research in Social Education, 1, vol. XVI, 69-82.

Todorov, $\mathrm{T}$.

2000 Los abusos de la memoria. Barcelona: Paidós.

Tutiaux-Guillon, N.; Mousseau, M.J.

1998 Les jeunes et l'histoire. Identités, valeurs, conscience historique. París: INRP.

Whitrow,G.J.

1990 El tiempo en la historia. Barcelona: Crítica.

Wineburg, S.S.

1996 The psichology of learning and teaching history. Berliner, D.; Calfee, R.C. (ed.). Handbook of Educational Psichology. New York: New York University. 423-437.

Wineburg, S.S.

2000 Making Historical Sense. Stearns, P.N.; Seixas, P.; Wineburg, S.S. (eds.) Knowing, Teaching, and Learning History. New York: New York University Press. 306-325.

Zaccaria, M.A.

1980 The development of historical thinking: inplications for the teaching of history. The History Teacher, 3, 323-340. En castellà a Pereyra, M. (ed.) La historia en el aula. Santa Cruz de Tenerife: ICE-Universidad de la Laguna, 1982. 191-207. 


\section{Nota}

$1 \quad$ Investigaciones del grupo GREDICS (Grup de Recerca en Didàctica de les Ciències Socials) de la Universidad
Autónoma de Barcelona, Grupo de Investigación Reconocido (2014 SGR 1384). 
